\title{
Unusual Synchronous Association of Solid Tumors with Hematological Malignancies in Multiple Primary Cancers: Case Series and Literature Review
}

\author{
Aboelkhair Al-Gahmi ${ }^{a}$ Mohammed Alhuthali ${ }^{b} \quad$ Mohammad Alrehailia \\ Badee Baltow ${ }^{\mathrm{c}}$ Emad Tashkandi ${ }^{\mathrm{a}}$ b \\ aDepartment of Medical Oncology, Oncology Center, King Abdullah Medical City, Makkah, \\ Saudi Arabia; ${ }^{b}$ College of Medicine, Umm Al-Qura University, Makkah, Saudi Arabia; \\ 'Laboratory and Blood Bank, King Abdullah Medical City, Makkah, Saudi Arabia
}

\author{
Keywords \\ Synchronous malignancies · Solid tumor · Hematological malignancy · Multiple primary \\ malignancy
}

\begin{abstract}
The incidence of synchronous multiple primary malignancies (MPMs), which include both solid and hematological malignancy, is considered very rare. In addition, the involvement of sites such as brain, thyroid, and breast are among the least reported in such complex conditions. Here we report five different types of solid tumors including glioblastoma multiforme, thyroid papillary carcinoma, breast invasive ductal carcinoma, colon cancer, and gastric adenocarcinoma that were associated with synchronous five different hematological malignancies in the form of T-cell lymphoblastic non-Hodgkin lymphoma (NHL), nodal marginal zone $\mathrm{NHL}$, diffuse large B-cell NHL, Hodgkin lymphoma, and gastric mucosa-associated lymphoid tissue marginal zone NHL, respectively. The diagnosis of MPMs can be challenging, and there is no standard treatment for such difficult primary malignancies. However, the management of these conditions should be individualized using tumor board discussion and ensuring multidisciplinary coordinated care, besides considering treatment of the more aggressive malignancy before that with the less malignant potential.


Al-Gahmi et al.: Synchronous Multiple Primary Malignancies

\section{Introduction}

Multiple primary malignancies (MPMs) in a single individual is considered a well-established phenomenon with a reported prevalence ranging between 0.73 and $11.7 \%$ [1]. However, synchronous category of MPMs that is found either simultaneously or within 6 months of the diagnosis of the first primary malignancy is considered less common $[2,3]$. Although multiple risk factors such as genetic predisposition, immunosuppression, smoking, chemotherapy, and ionizing radiation are thought to contribute to the development of this condition, the exact pathophysiology remains not clearly understood [2-4].

In our series, we present 5 cases of synchronous double primary malignancies consisting of a combination of solid tumor and hematological malignancy. The five different types of solid tumors include brain glioblastoma multiforme, thyroid papillary carcinoma, breast invasive ductal cancer, colon cancer, and gastric adenocarcinoma, and they were associated with T-cell lymphoblastic non-Hodgkin lymphoma (NHL), nodal marginal zone NHL, diffuse large B-cell (DLBC) NHL, Hodgkin lymphoma, and gastric mucosa-associated lymphoid tissue (MALT) marginal zone NHL, respectively. We felt that our cases are worth reporting as the synchronous association of solid tumors with a hematological malignancy like Hodgkin disease or NHL is unusual and still little reported [2, 3, 5, 6]. Moreover, locations such as brain, thyroid, and breast are very rare in such synchronous MPMs [3, 5].

\section{Case Series}

Five patients were transferred to the medical oncology department at King Abdullah Medical City in Makkah, Saudi Arabia for oncologic evaluation of their different medical conditions. Workup revealed synchronous double malignancies characterized by a combination of solid tumor associated with hematological malignancy. A summary of the patient's clinical data is shown in Table 1, with further details found under each case description.

\section{Case 1}

A 15-year-old male had been complaining of a headache and double vision with nausea for 3 months. Brain magnetic resonance imaging was performed at an outside facility and revealed a left parieto-occipital space-occupying lesion with a large cystic component (Fig. 1). He had craniotomy done with subtotal excision and the pathology was consistent with glioblastoma multiforme (Fig. 2).

Table 1. Summary of the cases with double primary malignancies

\begin{tabular}{|c|c|c|c|c|c|c|}
\hline \multirow[t]{2}{*}{ Number } & \multirow[t]{2}{*}{ Sex } & \multirow{2}{*}{$\begin{array}{l}\text { Age, } \\
\text { years }\end{array}$} & \multicolumn{2}{|l|}{ Solid malignancy } & \multicolumn{2}{|l|}{ Hematological malignancy } \\
\hline & & & diagnosis & treatment & diagnosis & treatment \\
\hline 1 & M & 15 & glioblastoma multiforme & surgery & T-lymphoblastic NHL & chemotherapy \\
\hline 2 & $\mathrm{~F}$ & 82 & thyroid papillary cancer & palliative & nodal marginal zone NHL & conservative \\
\hline 3 & $\mathrm{~F}$ & 71 & breast IDC & surgery + hormonal & DLBC NHL & chemotherapy \\
\hline 4 & $\mathrm{~F}$ & 48 & colon adenocarcinoma & surgery & Hodgkin’s lymphoma & chemotherapy \\
\hline 5 & M & 53 & gastric cancer & chemotherapy & gastric MALT, marginal zone NHL & conservative \\
\hline
\end{tabular}

DLBC, diffuse large B-cell; F, female; IDC, invasive ductal carcinoma; M, male; MALT, mucosa-associated lymphoid tissue; NHL, non-Hodgkin lymphoma. 


\section{Case Reports in Oncology}

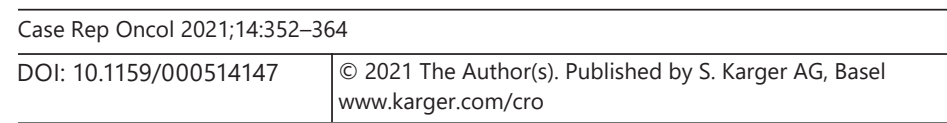

Al-Gahmi et al.: Synchronous Multiple Primary Malignancies

Fig. 1. Brain magnetic resonance imaging with contrast shows a left parieto-occipital space-occupying lesion with a large cystic component.

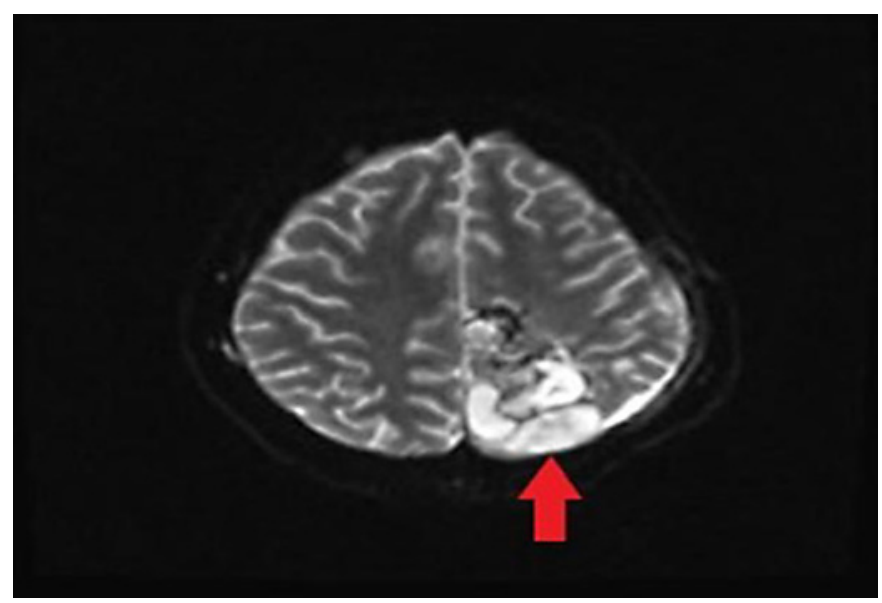

Fig. 2. Large multinucleated and pleomorphic cells consistent with glioblastoma multiforme.

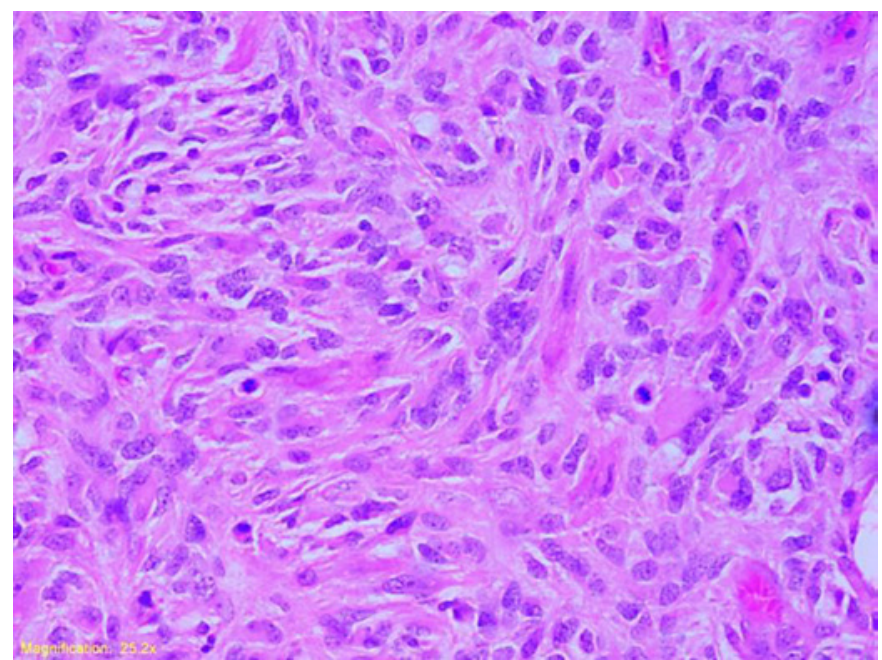

A few weeks later, his condition was complicated by the development of dyspnea for which chest X-ray showed massive right pleural effusion. A computed tomography (CT) scan of the chest showed a mediastinal mass (Fig. 3), and histopathology was consistent with T-cell lymphoblastic NHL (Fig. 4). Immunohistochemical staining revealed that the tumor cells were positive for CD3 and TdT, while they were negative for CD79a, CD30, BCL-2, and cyclin D1.

The case was discussed in the tumor board and he was planned to receive temozolomide with concurrent radiation therapy to the residual lesion in the brain after the end of the treatment of his aggressive lymphoma. As the patient was found not to be fit for highly toxic chemotherapy regimens such as hyper-CVAD (cyclophosphamide, vincristine, doxorubicin, and dexamethasone) alternating with high-dose methotrexate and cytarabine, the decision was taken to administer a dose-dense CHOP regimen every 2 weeks with filgrastim for eight cycles on an outpatient basis. However, he only received six cycles of CHOP with good initial response which was followed by rapid progression, and he died before receiving adjuvant treatment for his brain tumor.

\section{Case 2}

An 82-year-old female patient presented with long-standing left cervical swelling. CT of the neck showed large heterogeneous enhancement of the soft tissue mass seen in the left supraclavicular region, measuring about $4.6 \times 5.0 \times 6.4 \mathrm{~cm}$ (Fig. 5). Internal hypodensity was 


\section{Case Reports in Oncology}

Fig. 3. Computed tomography of the chest showing huge mediastinal swelling.

Fig. 4. Mediastinal T-lymphoblastic non-Hodgkin lymphoma. Medium magnification view shows sheets of small to medium-sized lymphoid cells with condensed nuclear chromatin and numerous mitoses.

Fig. 5. Computed tomography of the neck with contrast shows a large heterogeneous enhancement of the soft tissue mass seen in the left supraclavicular region, measuring about $4.6 \times 5.0 \times 6.4$ $\mathrm{cm}$. Internal hypodensity was noted within the lesion suggestive of necrosis. There were bilateral thyroid nodules, some of which were partially calcified, with left thyroid lobe enlargement and heterogeneous enhancement.

\begin{tabular}{l|l}
\hline Case Rep Oncol 2021;14:352-364 \\
\hline DOI: 10.1159/000514147 & $\begin{array}{l}\text { ○ 2021 The Author(s). Published by S. Karger AG, Basel } \\
\text { www.karger.com/cro }\end{array}$ \\
\hline
\end{tabular}
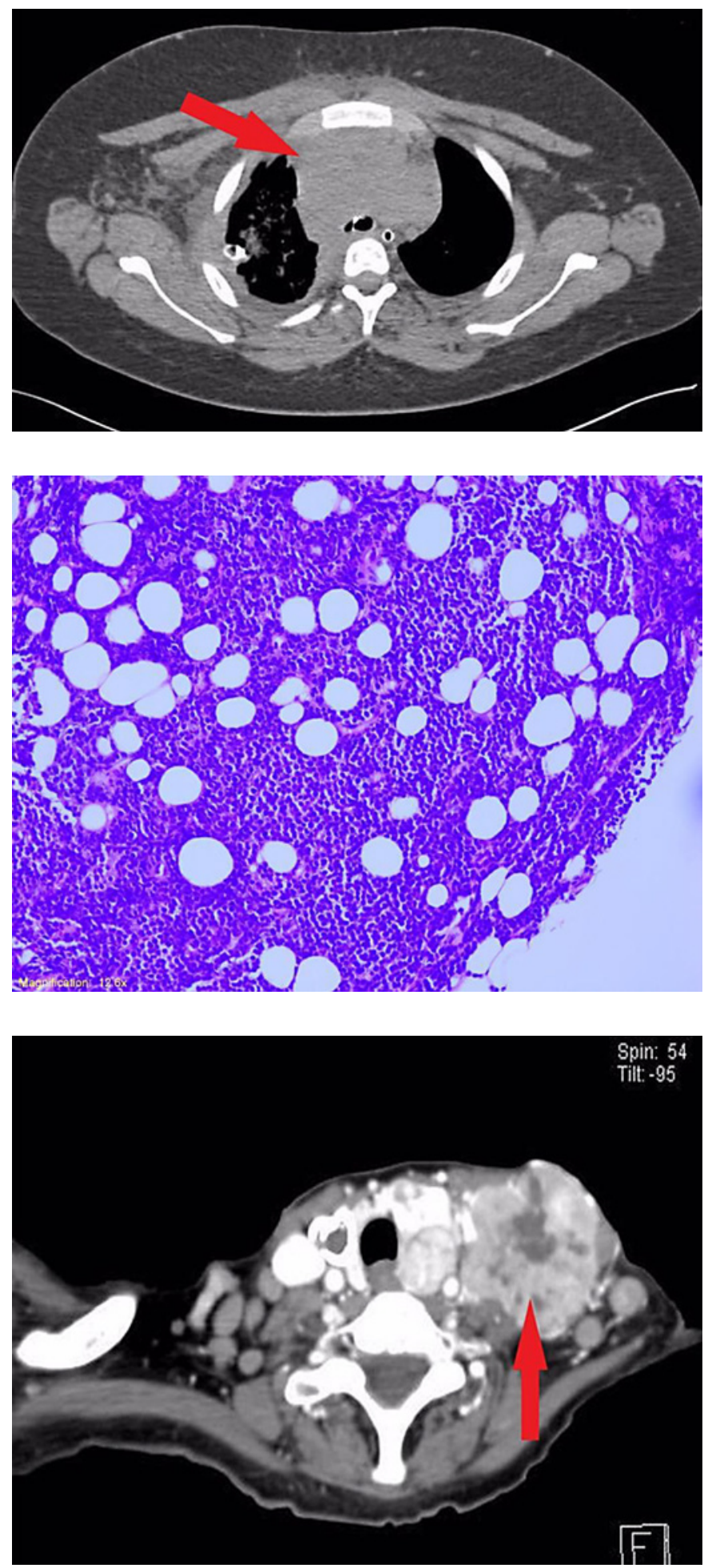

noted within the lesion, suggestive of necrosis. The mass extended to the skin and was inseparable from the ipsilateral sternocleidomastoid muscle. There were bilateral thyroid nodules, some of which were partially calcified, with left thyroid lobe enlargement and heterogeneous enhancement. Bilateral parotid and submandibular glands appeared unremarkable. 


\section{Case Reports in Oncology}

Fig. 6. Computed tomography of the abdomen revealed multiple matted retroperitoneal lymph nodes surrounding the aorta, inferior vena cava, and iliac vessels.

Fig. 7. Nodal marginal zone nonHodgkin lymphoma histopathology showing infiltration of small to medium-sized B lymphocytes that express positivity for CD20 (strong and diffuse), CD43, and BCL-2.

Fig. 8. Fine needle aspiration of the thyroid showing papillary architecture and tumor cells with finely dispersed chromatin consistent with papillary thyroid cancer.

\begin{tabular}{l|l}
\hline Case Rep Oncol 2021;14:352-364 \\
\hline DOI: 10.1159/000514147 & $\begin{array}{l}\text { ○ 2021 The Author(s). Published by S. Karger AG, Basel } \\
\text { www.karger.com/cro }\end{array}$ \\
\hline
\end{tabular}
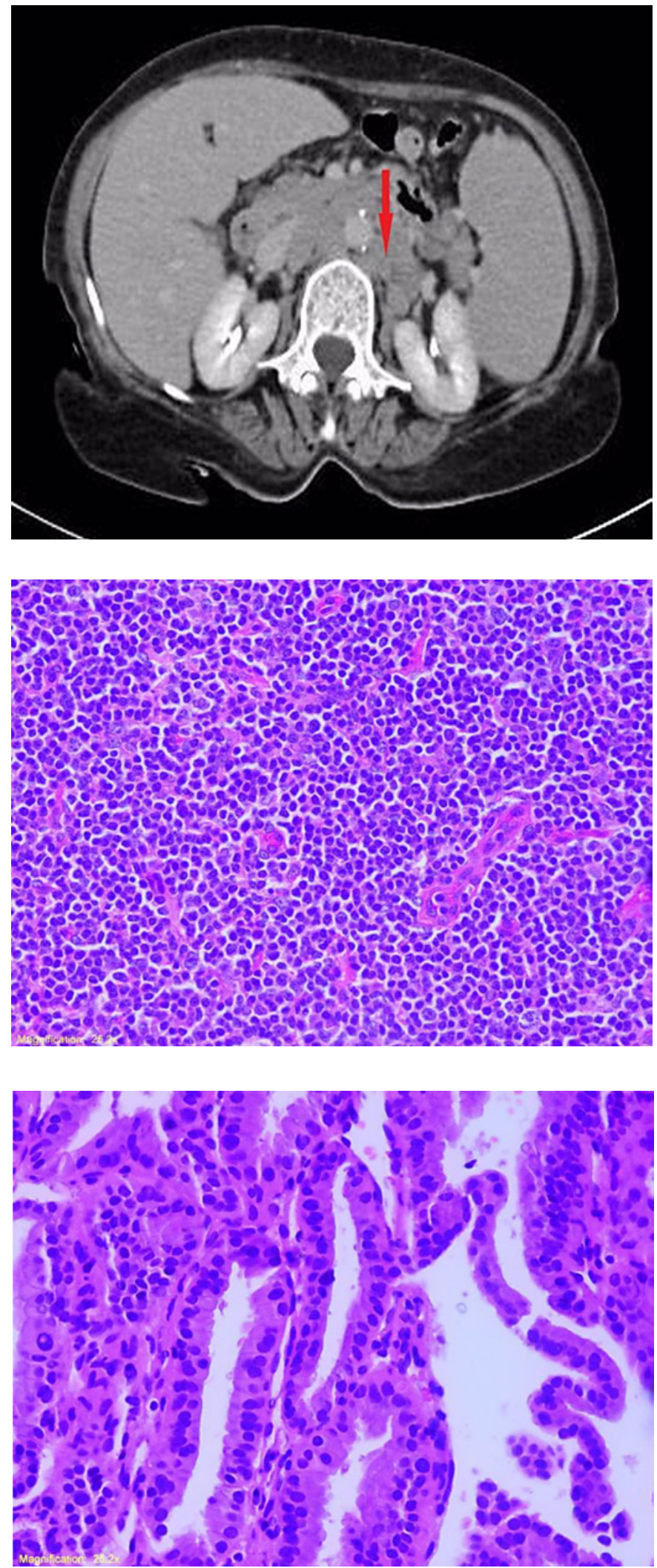


\section{Case Reports in Oncology}

Fig. 9. Breast mass biopsy shows invasive ductal carcinoma. Tubular formation and nest-like structures are seen in this low-magnification image.

Fig. 10. Ultrasound of the right breast revealed a small irregular hypoechoic mass measuring approximately $2.4 \times 1.0 \mathrm{~cm}$ with speculated outlines.

\begin{tabular}{l|l}
\hline Case Rep Oncol 2021;14:352-364 \\
\hline DOI: 10.1159/000514147 & $\begin{array}{l}\text { ○ 2021 The Author(s). Published by S. Karger AG, Basel } \\
\text { www.karger.com/cro }\end{array}$ \\
\hline
\end{tabular}
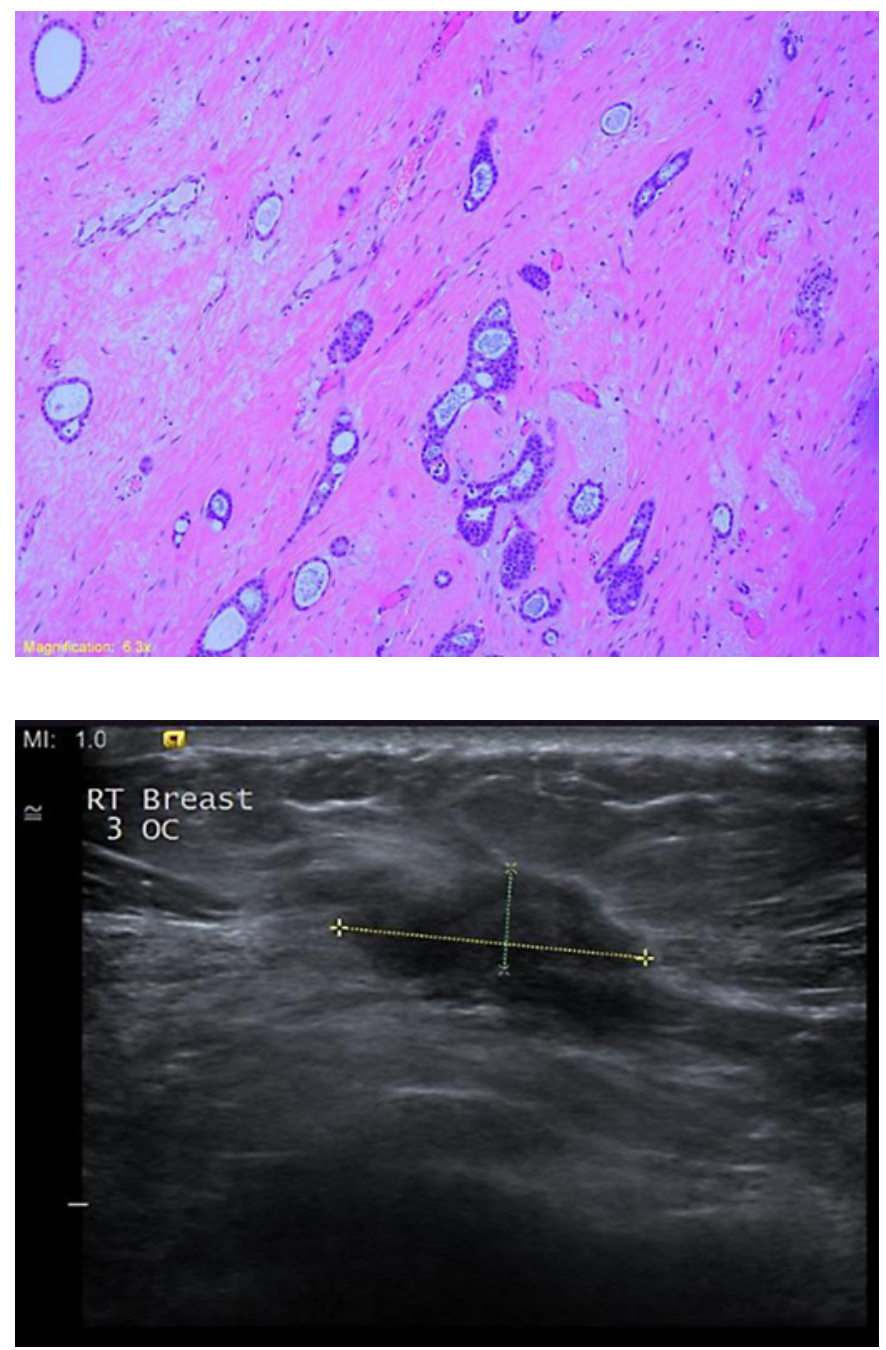

CT of the abdomen revealed multiple matted retroperitoneal lymph nodes surrounding the aorta, inferior vena cava, and iliac vessels (Fig. 6). The largest lymph node was in the leftside para-aortic group, measuring $1.4 \times 3.3 \mathrm{~cm}$. There was also a $5.3 \times 6.5 \mathrm{~cm}$ right pelvic external iliac lymph node besides bilateral enlarged inguinal lymph nodes, with the largest on the right measuring $2.7 \times 1.3 \mathrm{~cm}$.

Left cervical and ultrasound-guided abdominal lymph node histopathology results were compatible with nodal marginal zone NHL (Fig. 7) which showed positivity for CD20 (strong and diffuse), CD43, and BCL-2. However, left thyroid fine needle aspiration revealed a second diagnosis of malignant papillary cancer (Fig. 8).

The patient was not fit for surgery and instead was treated with symptomatic treatment as she had poor performance status. We maintained a watchful waiting approach for her marginal zone lymphoma. Finally, the patient's code status was made "do not resuscitate" and then she was transferred to palliative care for follow-up.

Case 3

A 71-year-old woman was diagnosed with right breast cancer at an outside facility and referred to our oncology center for further evaluation and treatment. Histopathology review from her image-guided biopsy was consistent with grade 1 invasive ductal carcinoma (IDC) 


\section{Case Reports in Oncology}

Fig. 11. Diffuse proliferation of deeply staining large to mediumsized lymphoid cells that are consistent with a diagnosis of diffuse large B-cell non-Hodgkin lymphoma.

Fig. 12. A computed tomography scan revealed a few enlarged left cervical lymph nodes, with the largest one measuring approximately $2.0 \times 1.5 \mathrm{~cm}$

\begin{tabular}{l|l}
\hline Case Rep Oncol 2021;14:352-364 \\
\hline DOI: 10.1159/000514147 & $\begin{array}{l}\text { ○ 2021 The Author(s). Published by S. Karger AG, Basel } \\
\text { www.karger.com/cro }\end{array}$ \\
\hline
\end{tabular}
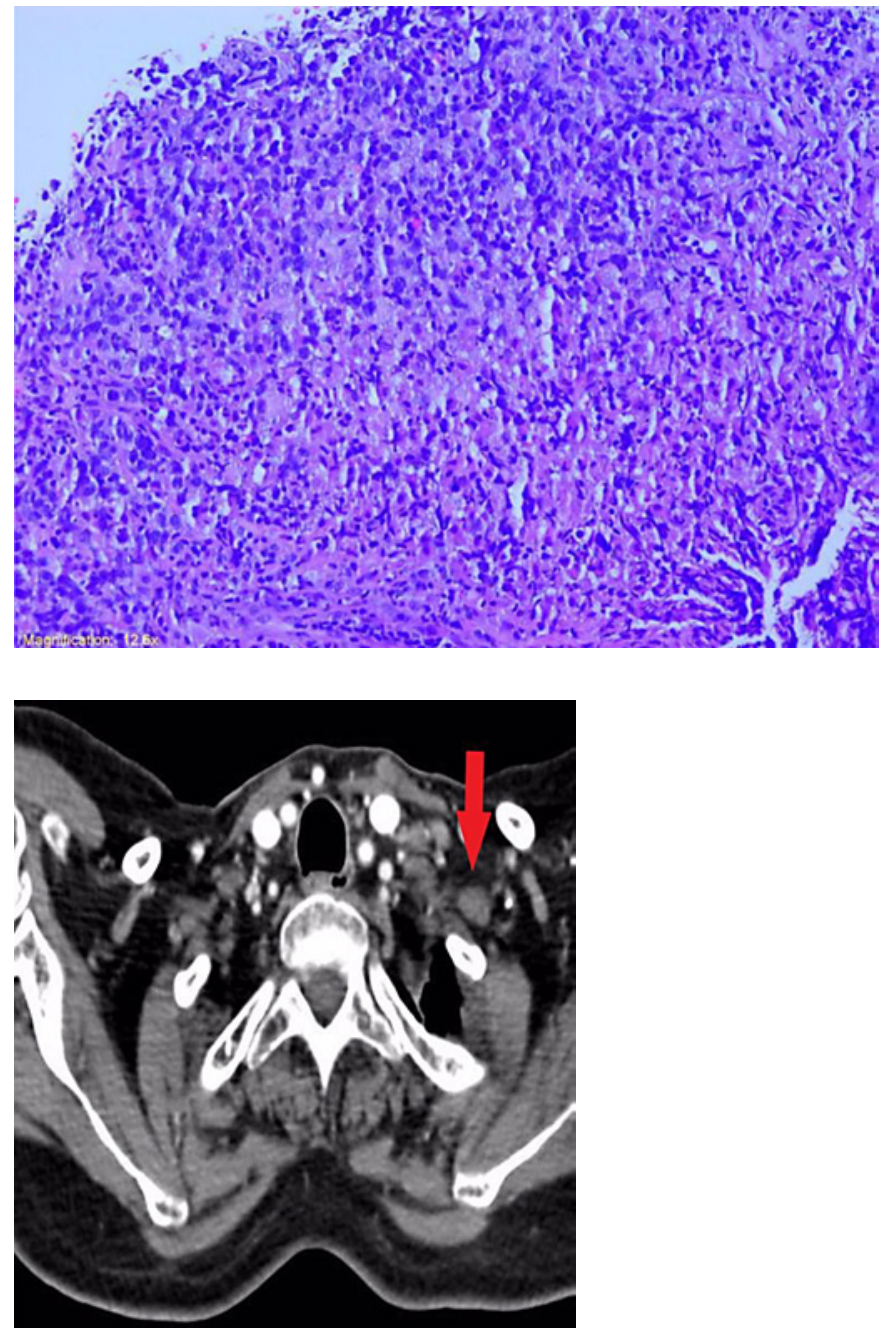

(Fig. 9) with a receptor status of ER-positive (100\%) and PR-positive (80\%), but negative for HER-2. Ultrasound of the breasts revealed a small irregular hypoechoic mass measuring approximately $2.4 \times 1.0 \mathrm{~cm}$ with speculated outlines located in the right breast at approximately 3 o'clock position (Fig. 10). Multiple benign-looking left axillary lymph nodes were noted and the metastatic workup including CT scans was negative. The patient was started on letrozole in addition to calcium and vitamin D, with acceptable tolerance and good clinical response.

During follow-up, she was found to have a small left cervical lymph node. The patient was feeling at her baseline at that point; however, a biopsy was done for further assessment as breast cancer rarely metastasizes to cervical lymph nodes and the results were consistent with DLBC NHL (Fig. 11). A CT scan revealed a few enlarged left cervical lymph nodes, with the largest one measuring approximately $2.0 \times 1.5 \mathrm{~cm}$ (Fig. 12), and borderline-sized retroperitoneal lymph nodes. No suspicious osseous or visceral focal lesions were observed. Bone marrow biopsy was negative and positron emission tomography CT scan revealed a right breast ill-defined lesion with a standardized uptake value of 1.7 and left lower cervical and para-aortic retroperitoneal lymph nodes with a standardized uptake value of 3 .

The case was discussed in the lymphoma and breast board and was planned for R-CHOP consisting of rituximab, cyclophosphamide, doxorubicin hydrochloride, vincristine, and prednisone, followed by breast-conserving surgery if good clinical response to the chemo- 


\section{Case Reports in Oncology}

\begin{tabular}{l|l}
\hline Case Rep Oncol 2021;14:352-364 \\
\hline DOI: 10.1159/000514147 & $\begin{array}{l}\text { ○ 2021 The Author(s). Published by S. Karger AG, Basel } \\
\text { www.karger.com/cro }\end{array}$ \\
\hline
\end{tabular}

Al-Gahmi et al.: Synchronous Multiple Primary Malignancies

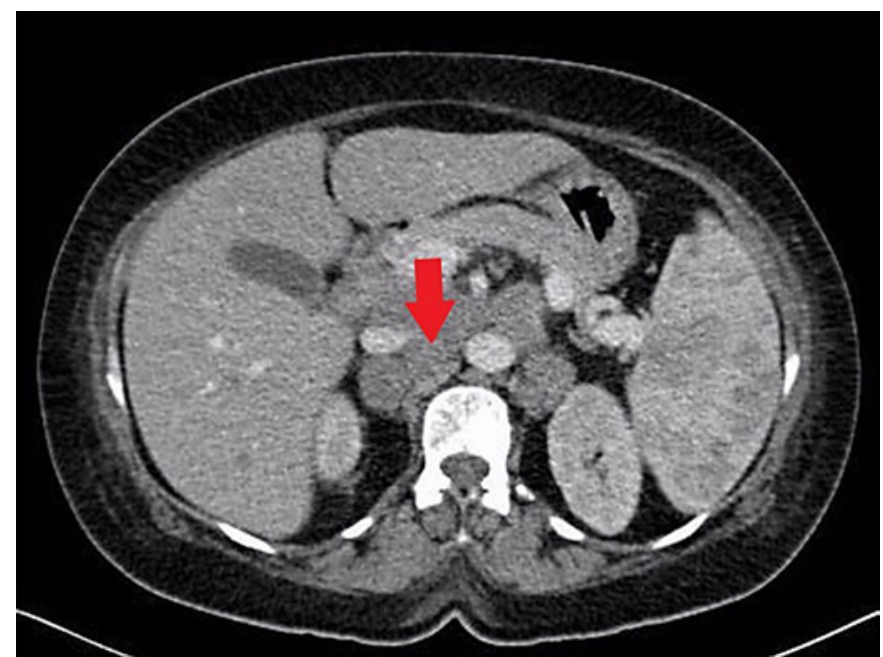

Fig. 13. Computed tomography scan of the abdomen showing retroperitoneal lymphadenopathy (arrow), splenomegaly, splenic and hepatic lesions, and sclerotic bone lesions of the first lumbar vertebra.

therapy occurred. The patient received one cycle of R-CHOP with poor tolerance. Thus, she was switched to R-CVP (rituximab, cyclophosphamide, vincristine, and prednisone) on the subsequent cycles with good tolerance, and letrozole was continued. A repeat CT scan revealed almost complete resolution of the previously noted lymphadenopathy.

After completing four cycles of chemotherapy, the patient had a lumpectomy and axillary lymph node dissection, and pathology was consistent with the previous histopathological diagnosis of grade 1 IDC. The size of the tumor was $1.4 \times 1.0 \times 0.8 \mathrm{~cm}$ with negative margins. No lymphovascular or perineural invasion was seen. Seventeen lymph nodes were sampled and all were found to be negative for tumor metastasis. Later on, she received adjuvant radiation therapy and was administered two cycles of rituximab with a plan to continue her letrozole for a minimum of 5 years. Upon follow-up in the next 2 years, the patient was found to be free of recurrence, with good health status.

\section{Case 4}

A 48-year-old woman with a history of ulcerative colitis for 5 years was admitted to our hospital with high-grade fever of $40^{\circ} \mathrm{C}$ associated with significant weight loss and night sweats for a few months. Routine laboratory workup was remarkable for a hemoglobin level of $6.5 \mathrm{~g} / \mathrm{dL}$ and platelets at $59 \times 10^{9} / \mathrm{L}$. CT revealed retroperitoneal lymphadenopathy, splenomegaly, splenic and hepatic lesions, and sclerotic bone lesions of the first lumbar vertebra (Fig. 13). She then had CT-guided biopsy done from the para-aortic lymph node and spinal lesion, and both were positive for lymphocyte-depleted classical Hodgkin lymphoma (Fig. 14). Properly controlled immunohistochemical staining showed that the neoplastic cells were positive for CD15, CD30, and Fascin, while they were negative for CD34, CD3, CD45, CD79a, CD138, and CK AE1/AE3.

The patient was discussed in the lymphoma and gastrointestinal tumor board and was planned to start on ABVD chemotherapy consisting of adriamycin, bleomycin, vinblastine, and dacarbazine. After one cycle she was admitted with intestinal obstruction and underwent subtotal colectomy with ileostomy.

The surgical pathology specimen was consistent with high-grade poorly differentiated adenocarcinoma (Fig. 15), and 16 lymph nodes out of 31 were positive for metastatic carcinoma. No adjuvant treatment was administered for colon cancer as the patient continued the treatment for three more cycles of ABVD, which unfortunately was followed by disease progression. Therefore, the treatment regimen was shifted to gemcitabine and cisplatin for 


\section{Case Reports in Oncology}

Fig. 14. Retroperitoneal lymph node biopsy revealing Hodgkin lymphoma, lymphocyte-depleted type. Highly anaplastic, large, pleomorphic cells that seem to resemble Reed-Sternberg cells are seen.

Fig. 15. Adenocarcinoma of the colon showing poorly differentiated malignant glandular structures.

\begin{tabular}{l|l}
\hline Case Rep Oncol 2021;14:352-364 \\
\hline DOI: 10.1159/000514147 & $\begin{array}{l}\text { @ 2021 The Author(s). Published by S. Karger AG, Basel } \\
\text { www.karger.com/cro }\end{array}$ \\
\hline
\end{tabular}
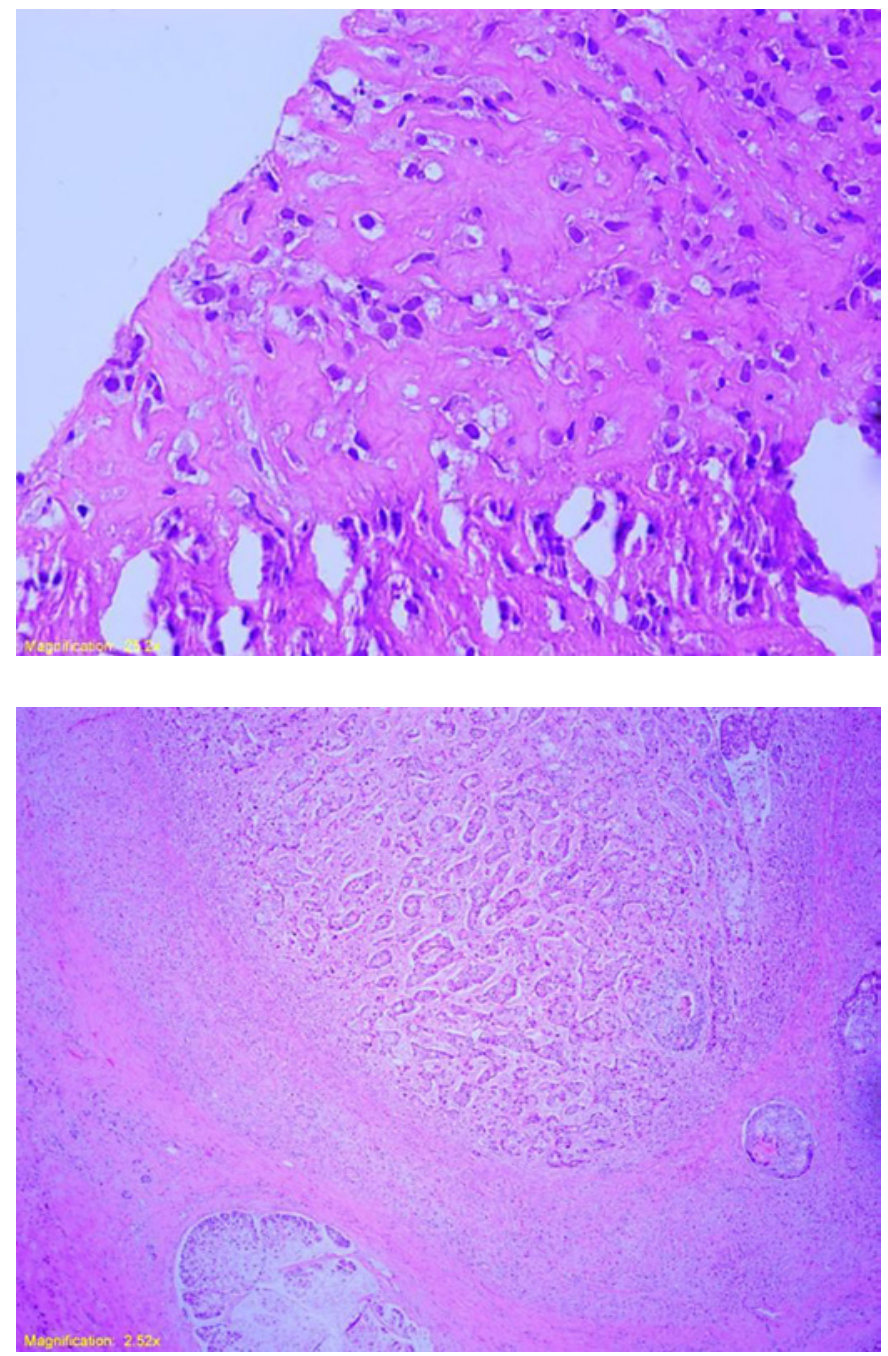

four cycles. However, the disease further progressed and her performance status became poor based on the Eastern Cooperative Oncology Group scale. Ultimately, her code status was made "do not resuscitate" and she was transferred to palliative care for symptomatic management before dying within the next few months.

\section{Case 5}

A 53-year-old male was admitted to our hospital with abdominal pain, constipation, and significant weight loss. CT of the abdomen (Fig. 16) showed irregular wall thickening in the stomach and duodenum and multiple variable-sized lymph nodes in the abdomen. Multiple enhancing heterogeneous liver lesions measuring $>2 \mathrm{~cm}$ and peritoneal metastases were also detected. An endoscopic biopsy of the stomach (Fig. 17) showed findings suggestive of well to moderately differentiated adenocarcinoma with a possibility of second tumor (collision tumor). Colonoscopy showed a $3.5-\mathrm{cm}$ rectal mass and the biopsy result showed moderately differentiated infiltrating adenocarcinoma. Properly controlled immunohistochemical staining showed neoplastic cell positivity for CK7 and CK20 in the gastric biopsy. CK7 was also positive in the colonic biopsy, while CK20 was negative. This staining pattern associated with the described morphology indicated that the adenocarcinoma was of gastric origin, with metastasis to the colon. 


\section{Case Reports in Oncology}

Fig. 16. Computed tomography of the abdomen showed irregular wall thickening in the stomach and duodenum (arrow) and multiple variable-sized lymph nodes in the abdomen. Multiple enhancing heterogeneous liver lesions measuring $>2 \mathrm{~cm}$ were also detected.

Fig. 17. Gastric biopsy showing adenocarcinoma component of a suspected collision malignancy.

Fig. 18. Gastric mucosa-associated lymphoid tissue marginal zone non-Hodgkin lymphoma characterized by monotonous proliferation of atypical lymphoid cells that appeared centrocyte-like, medium in size, and had round small to ovoid, hyperchromatic nuclei and little pale eosinophilic to clear cytoplasm.

\begin{tabular}{l|l}
\hline Case Rep Oncol 2021;14:352-364 \\
\hline DOI: 10.1159/000514147 & $\begin{array}{l}\text { ○ 2021 The Author(s). Published by S. Karger AG, Basel } \\
\text { www.karger.com/cro }\end{array}$ \\
\hline
\end{tabular}
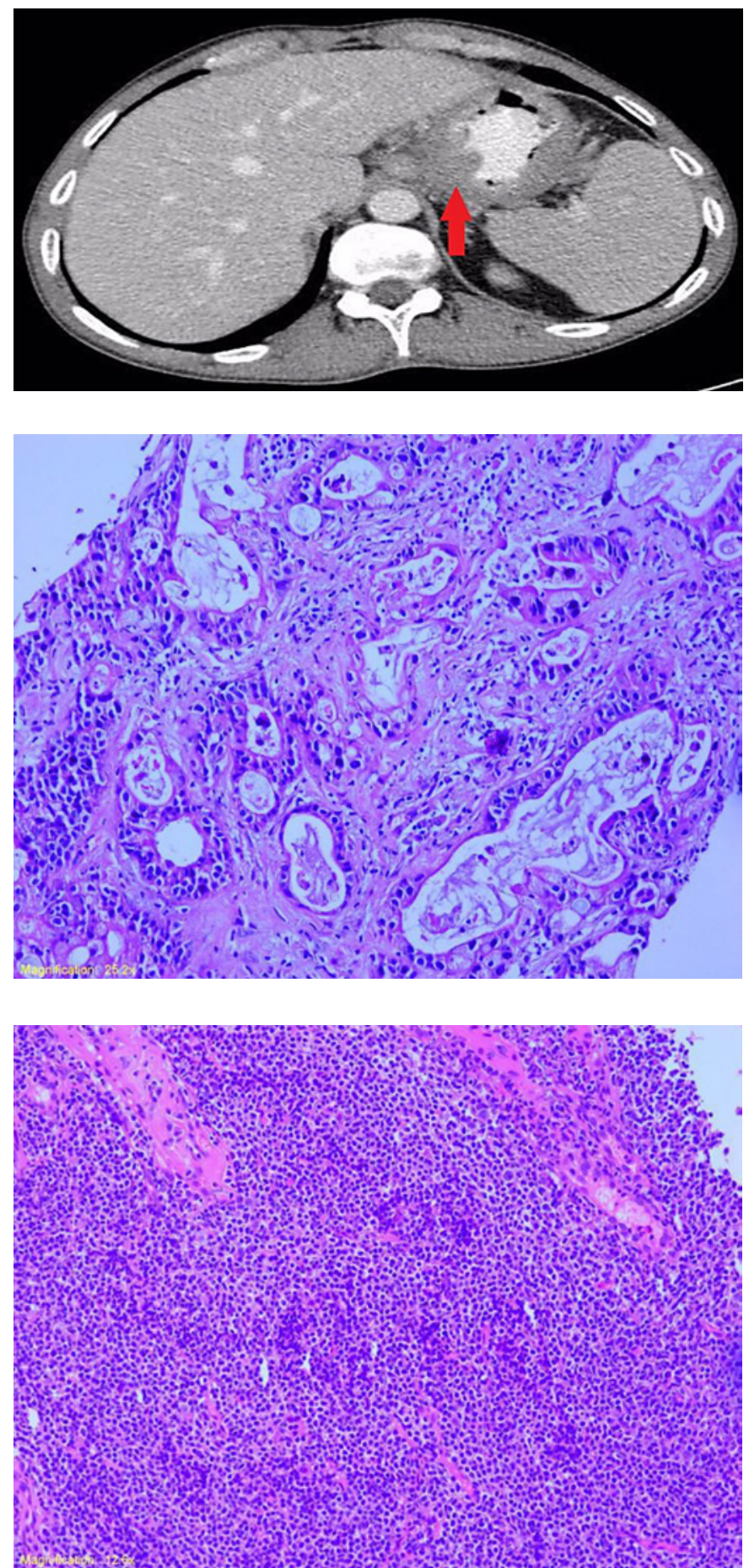

Subsequently, the patient was started on palliative modified FOLFOX protocol (folinic acid, fluorouracil, and oxaliplatin) with good response. However, he developed epigastric pain later on for which he had another gastroscopy with biopsy. Helicobacter pylori testing was negative but histopathology revealed monotonous proliferation of atypical lymphoid cells that appeared centrocyte-like, medium in size, and had round small to ovoid, hyperchromatic nuclei and little pale eosinophilic to clear cytoplasm (Fig. 18). In a few areas, small 
spared lymphoid follicles were seen with patent dendritic cell meshwork. Immunohistochemical stainings showed strong and diffuse positivity for CD20 and BCL-2 in atypical lymphoid cells, $2 \%$ of which showed proliferative activity as per Ki-67 reactivity. Morphological features and immunohistochemical profile favored the diagnosis of gastric MALT marginal zone NHL that was treated using conservative management including proton pump inhibitor. The patient is currently being observed with regular outpatient follow-up.

\section{Discussion}

MPMs represent a well-established phenomenon that has been frequently described in the medical literature [1]. Two categories have been recognized based on the timing of coexistence of these malignant tumors: Synchronous MPMs occur simultaneously or within 6 months of the diagnosis of first malignancy, while metasynchronous tumors accompany each other with longer intervals between each diagnosis [2]. Synchronous category, our focus in this study, is considered less common, with a prevalence rate ranging between 30 and $55 \%$ of all MPMs $[2,3,5]$.

Multiple pathology types have been identified among synchronous MPMs. Lv et al. [3] in their observational study found that adenocarcinomas (55.1\%) and squamous cell carcinomas $(23.1 \%)$ were the most common types, while sarcomas $(1.3 \%)$ and hematological malignancies including leukemias and lymphomas (6.4\%) were among the least frequently reported. Any organs can be involved in such conditions, but tumors affecting the gastrointestinal tract (from esophagus to rectum) and lungs, followed by involvement of the prostate, bladder, and kidney, are considered the most usual sites [3, 7-10]. Locations including brain, thyroid, breast, and lymphoid tissues, as seen in this case series, are very rare in synchronous MPMs [3, 5]. Moreover, the synchronous association of solid tumor with a hematological malignancy is considered one of the rarest association among synchronous MPMs.

The significance of our case series can be seen from multiple dimensions. Firstly, a simultaneous coexistence of glioblastoma multiforme with T-cell lymphoblastic NHL is reported here for the first time. Both tumors share a high level of aggressiveness that contributed to the grave prognosis seen in our case. On the other hand, the type of nodal marginal zone NHL that was associated synchronously with thyroid papillary cancer has not been described previously [11]. Moreover, although the colon is a common site of involvement in synchronous MPMs, its association with retroperitoneal Hodgkin lymphoma has never been seen previously either according to our literature review. There were some reported cases of synchronous breast IDC and DLBC NHL [12]; however, the successful treatment of our case based on our multidisciplinary coordination was unique. Finally, the rare but well-documented association between synchronous gastric adenocarcinoma and gastric MALT marginal zone NHL that is believed to be strongly associated with active $H$. pylori infection was not the case in our patient who tested negative for the infection [13]. Therefore, this case presentation should alert us to look for other possibly associated etiologies.

The mechanisms that are related to the development of synchronous MPMs are still not well explained. However, there are multiple etiologic factors that can contribute to the pathophysiology of such malignancies [2-4]. Genetic susceptibility and familial cancer syndromes are believed to be among these factors. Moreover, lifestyle-related exposures such as smoking, alcohol consumption, and some dietary patterns can play a significant role in the formation of a wide variety of multiple tumors. Immune deficiency states and previously administered treatments such as hormonal medications, chemotherapy, or radiation are also strongly associated with these conditions.

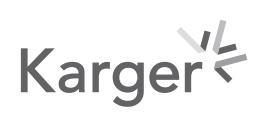


The diagnosis of synchronous MPMs can be difficult and challenging. Although it is unreasonable to get a biopsy for every metastatic lesion that may appear in cases like MPMs, a re-biopsy is the only option to confirm the possibility of MPMs. For example, the mediastinal lymphadenopathy that was described above in the case of glioblastoma multiforme is not a typical finding for such cases. Therefore, a decision of mediastinal lymph node biopsy was made to look for double malignancy. Also, cervical lymphadenopathy is uncommon in breast IDC; hence, a cervical lymph node biopsy was performed in our case which subsequently revealed DLBC NHL. Based on such observations, understanding the different malignant behaviors of solid tumors and hematological malignancies in addition to the ability to differentiate between them is crucial for selecting which lesion to biopsy and also pivotal before treating such complicated conditions.

Multiple treatment modalities can be offered including surgery, chemotherapy, and radiation treatment, but there are no available guidelines in the management of such diseases [2, 5]. However, it is common practice to deal with synchronous MPMs as independent malignancies and start treatment of the most aggressive cancer rather than to treat the tumor with the least malignant potential $[14,15]$. Simultaneous surgeries for more than one malignancy can be considered in some carefully selected cases [14]. Based on our experience, we believe that the treatment plan should be determined using tumor board discussion, ensuring a multidisciplinary team approach and expertise and close attention to the features of the patient's disease and functional status. Our success in the treatment of previously described cases of synchronous breast IDC and DLBC NHL is one of the examples of how much multimodal coordination of care contributes to positive outcome.

Research has shown that synchronous MPMs are associated with a significant reduction in overall survival in comparison to metasynchronous malignant tumors [16]. Nevertheless, the prognosis of such medical conditions is very variable and mainly determined by the outcome of the most aggressive tumor [14].

\section{Conclusion}

Synchronous MPMs, including double primary malignancies involving locations such as brain, thyroid, breast, and lymphoid tissues, are considered very rare. Moreover, synchronous association between a solid tumor and hematological malignancy such as Hodgkin disease or NHL is also an unusual finding. A high index of suspicion should be used when dealing with such complex conditions. In addition, we should consider performing another biopsy if the clinical and radiological findings are not typical for the primary malignancy. A complete evaluation including preoperative assessment and interdisciplinary collaboration can always guarantee the best possible treatment for a patient.

\section{Acknowledgment}

We would like to thank the Department of Pathology at King Abdullah Medical City, Makkah, Saudi Arabia for their support with this paper.

\section{Statement of Ethics}

Written informed consent was obtained from each patient for publication of their case and any accompanying images.

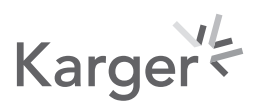




\section{Conflict of Interest Statement}

The authors have no conflicts of interest to declare.

\section{Funding Sources}

No funding was provided for this study.

\section{Author Contributions}

A. Al-Gahmi wrote the introduction and description of cases with some contribution from M. Alhuthali. M. Alrehaili wrote the discussion part and B. Baltow prepared the pathology images with their description. The entire paper was revised by E. Tashkandi and A. Al-Gahmi.

\section{References}

1 Demandante CG, Troyer DA, Miles TP. Multiple primary malignant neoplasms: case report and a comprehensive review of the literature. Am J Clin Oncol. 2003;26:79-83.

2 Cui Y, Liu T, Zhou Y, Ji Y, Hou Y, Jin W, et al. Five cases report of solid tumor synchronously with hematologic malignancy. Cancer Res Treat. 2012 Mar;44(1):63-8.

3 Lv M, Zhang X, Shen Y, Wang F, Yang J, Wang B, et al. Clinical analysis and prognosis of synchronous and metachronous multiple primary malignant tumors. Medicine (Baltimore). 2017;96(17):e6799.

4 Copur MS, Manapuram S. Multiple primary tumors over a lifetime. Oncology (Williston Park). 2019 Jul;33(7): 629384.

5 Liu S, Wei X, Xiong Y, Mi R, Yin Q. Thirty-two case reports of synchronous hematological malignancy and solid tumor. Turk J Haematol. 2019;36(4):291-4.

6 Comez G, Pehlivan Y, Kalender ME, Sevinc A, Sari I, Camci C. Synchronous Hodgkin's disease and gastric adenocarcinoma. Oncology. 2007;73(5-6):422-5.

7 Kilciksiz S, Gokce T, Baloglu A, Calli A, Kaynak C, Kilic B, et al. Characteristics of synchronous- and metachronous-type multiple primary neoplasms: a study of hospital-based cancer registry in Turkey. Clin Genitourin Cancer. 2007;5(7):438-45.

8 Aydiner A, Karadeniz A, Uygun K, Tas S, Tas F, Disci R, et al. Multiple primary neoplasms at a single institution: differences between synchronous and metachronous neoplasms. Am J Clin Oncol. 2000;23(4):364-70.

9 Liu Z, Liu C, Guo W, Li S, Bai O. Clinical analysis of 152 cases of multiple primary malignant tumors in 15,398 patients with malignant tumors. PLoS One. 2015;10(5):e0125754.

10 Li W, Zhan Y, Li G. Double cancers: a clinical analysis of 156 cases. Zhonghua Zhong Liu Za Zhi. 1996;18:296-8.

11 Popivanov GI, Bochev P, Hristoskova R, Mutafchiyski VM, Tabakov M, Philipov A, et al. Synchronous papillary thyroid cancer and non-Hodgkin lymphoma: case report. Medicine (Baltimore). 2018;97(6):e9831.

12 Woo EJ, Baugh AD, Ching K. Synchronous presentation of invasive ductal carcinoma and mantle cell lymphoma: a diagnostic challenge in menopausal patients. J Surg Case Rep. 2016;2016(1):rjv153.

13 Sonpal N, Mathur S, Arya M. Concurrent gastric MALT lymphoma and adenocarcinoma in a H. pylori negative patient: a rare finding with an even rarer presentation. Am J Gastroenterol. 2012 Oct;107:S296-S297.

14 Chowdary T, Sivaraj S, Rao G, Thirunavukkarasu S. Dual malignancies: do they have a worse prognosis than their individual counterparts. Arch Int Surg. 2015;5(1):29-32.

15 Singh A, Khare IC, Dixit AK, Pandey KC, Mittal DK, Singh P. Successfully treated synchronous double malignancy of the breast and esophagus: a case report. J Med Case Rep. 2010 Jun;4:169.

16 Skelton WP 4th, Ali A, Skelton MN, Federico R, Bosse R, Nguyen TC, et al. Analysis of overall survival in patients with multiple primary malignancies: a single-center experience. Cureus. 2019 Apr;11(4):e4552.

\section{Karger'}

